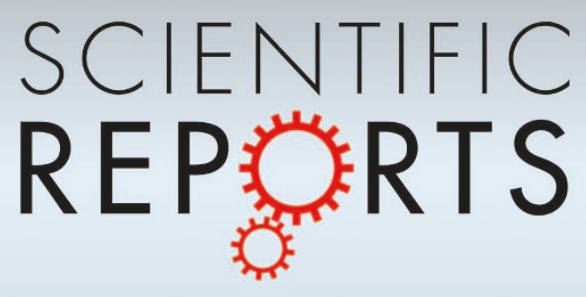

OPEN

SUBJECT AREAS:

SURFACES, INTERFACES

AND THIN FILMS

ELECTRONIC PROPERTIES AND

DEVICES

CHEMICAL ENGINEERING

ELECTRONIC PROPERTIES AND MATERIALS

Received

23 August 2012

Accepted

4 December 2012

Published

17 December 2012

Correspondence and requests for materials should be addressed to

H.H. (physhh@csu. edu.cn) or A.T.S.W. (phyweets@nus.edu.

$\mathrm{sg}$

\footnotetext{
* These authors contributed equally to
} this work.

\section{Spatially Resolved Electronic Structures of Atomically Precise Armchair Graphene Nanoribbons}

\author{
Han Huang ${ }^{1,2,3 *}$, Dacheng Wei ${ }^{1 *}$, Jiatao Sun ${ }^{4}$, Swee Liang Wong ${ }^{1,5}$, Yuan Ping Feng ${ }^{1}$, A. H. Castro Neto 1,2 \\ \& Andrew Thye Shen Wee ${ }^{1,2,5}$
}

\begin{abstract}
'Department of Physics, National University of Singapore, 2 Science Drive 3, Singapore, $117542,{ }^{2}$ Graphene Research Centre, National University of Singapore, Block S1 4, Level 6, 6 Science Drive 2, Singapore 1 17546, ${ }^{3}$ Institute of Super Microstructure and Ultrafast Process, School of Physics and Electronics, Central South University, No. 932 Lushan South Road, Changsha Hunan, People's Republic of China, 4 10083, ${ }^{4}$ Department of Chemistry, National University of Singapore, 3 Science Drive 3, Singapore, $117543,{ }^{5}$ NUS Graduate School for Integrative Sciences and Engineering, National University of Singapore, 28 Medical Drive, Singapore, 117456.
\end{abstract}

Graphene has attracted much interest in both academia and industry. The challenge of making it semiconducting is crucial for applications in electronic devices. A promising approach is to reduce its physical size down to the nanometer scale. Here, we present the surface-assisted bottom-up fabrication of atomically precise armchair graphene nanoribbons (AGNRs) with predefined widths, namely 7-, 14- and 21-AGNRs, on $\mathrm{Ag}(111)$ as well as their spatially resolved width-dependent electronic structures. STM/STS measurements reveal their associated electron scattering patterns and the energy gaps over $1 \mathrm{eV}$. The mechanism to form such AGNRs is addressed based on the observed intermediate products. Our results provide new insights into the local properties of AGNRs, and have implications for the understanding of their electrical properties and potential applications.

3 raphene, a single sheet of $\mathrm{sp}^{2}$-bonded carbon atoms arranged in a honeycomb lattice, exhibits extraordinary electrical and mechanical properties. For applications in electronic devices, graphene needs to be semiconducting. Reducing its physical size down to the nanometer scale is a promising approach. Theoretically, free-standing armchair graphene nanoribbons (AGNRs) can behave as either metals or semiconductors, depending on their widths ${ }^{1-3}$. GNRs fabricated by top-down methods generally possess uncontrolled and atomically imperfect edges, precluding detailed studies on finite size and edge effects ${ }^{4-6}$. Recently, the fabrication of 7-AGNRs (Fig. 1b, the number before "AGNR" indicates its width, that is, the number of carbon-pair lines in the direction perpendicular to AGNR axis, as sampled in Fig. 1d) from 10,10'-dibromo-9,9'bianthryl (DBBA, Fig. 1a) via polyanthracene has been demonstrated ${ }^{7,8}$. The GNR topology, width and edge periphery are predefined by the DBBA precursor molecule. Density functional theory (DFT) calculations suggest that dehydrogenation takes place in a domino-like fashion and is more favourable at sites between anthracene monomers than at their head sites. This limits the formation of wider AGNRs such as 14- and 21-AGNRs (Fig. 1c and 1d), which can be regarded as two and three 7-AGNRs conjugated side-by-side via dehydrogenation at the head sites, respectively.

Fabrication of atomically precise AGNRs with varying widths provides a unique opportunity to investigate quantum confinement and edge effects on them. In this article, we present the surface-assisted bottom-up fabrication of a series of atomically precise AGNRs from DBBA on $\operatorname{Ag}(111)$, such as 7-, 14- and 21-AGNRs, as well as their spatially resolved width-dependent electronic structures. Their typical vibration modes are measured by Raman spectroscopy. The associated electron scattering patterns (ESPs) which arise from intervalley electron backscattering at both armchair edges ${ }^{10,11}$ are visualized by scanning tunneling microscopy (STM). The local density of states (LDOS) on each AGNRs observed by scanning tunneling spectroscopy (STS) reveal the presence of energy gap over $1 \mathrm{eV}$. The mechanism to form such AGNRs is addressed based on the observed intermediate products. Our results are a significant step towards understanding the local properties of AGNRs, and provide us with design guidelines for the fabrication of edge-precise graphene nanoribbons for potential device applications. 


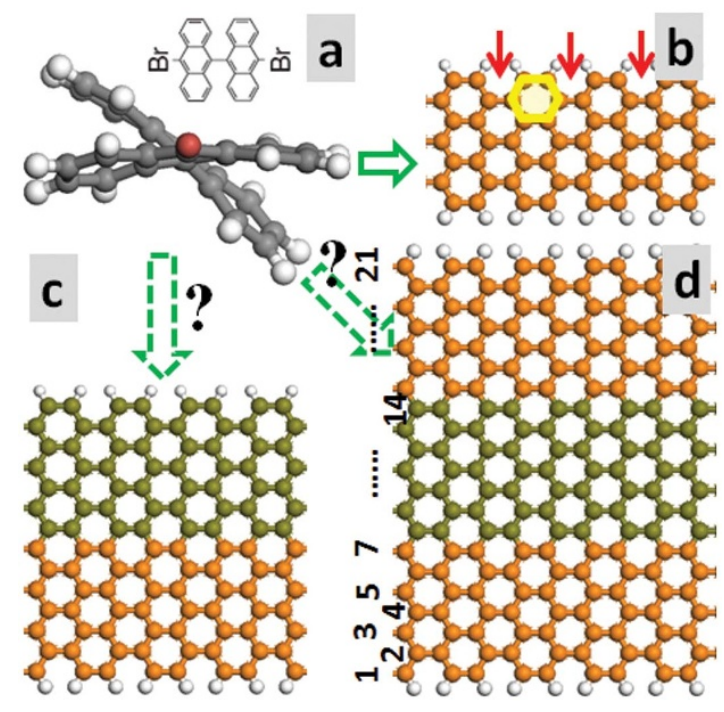

Figure 1 Schematic of surface-assisted bottom-up synthesis of atomically precise AGNRs from DBBA. a) side view of a ball-and-stick model of DBBA monomer, whose chemical structure is inserted; b) 7-AGNR; c) 14-AGNR; d) 21-AGNR. The number before "AGNR" indicates its width, that is, the number of carbon-pair lines in the direction perpendicular to AGNR axis, as sampled in Fig. 1d. In Fig. 1b, the yellow hexagon highlights an edged benzene-ring, the red arrows for the location between two benzene-rings edges.

\section{Results}

To provide a general characterization of the prepared AGNRs/ $\operatorname{Ag}(111)$ sample, ex situ Raman measurements were carried out at ambient atmosphere. Fig. 2a shows an average Raman spectrum from ten spots. Six representative modes in the range from $\sim 350 \mathrm{~cm}^{-1}$ to $\sim 1700 \mathrm{~cm}^{-1}$ are observed and identified as $G$ $\left(\sim 1610 \mathrm{~cm}^{-1}\right), \quad \mathrm{D} \quad\left(\sim 1350 \mathrm{~cm}^{-1}\right)$, the edge-phonon modes $\left(\sim 1220 \mathrm{~cm}^{-1}\right.$ and $\left.\sim 1260 \mathrm{~cm}^{-1}\right)$ and radial-breathing-like-mode (RBLM) $\left(\sim 396 \mathrm{~cm}^{-1}\right)$ as well as its third overtone RBLM3 $\left(\sim 960 \mathrm{~cm}^{-1}\right)^{12}$. Remarkably, the intensity ratio between modes D and $\mathrm{G}\left(I_{\mathrm{D}} / I_{\mathrm{G}}\right)$ is $\sim 0.90$, which is about twice that of AGNRs on $\mathrm{Au}(111)^{7}$. (also refer to Fig. S1) For polycrystalline graphite, the $I_{\mathrm{D}} / I_{\mathrm{G}}$ ratio correlates with the effective crystallite size $(\mathrm{La})$ as $\mathrm{La}^{2}$ for $L \mathrm{a}<2 \mathrm{~nm}$ : due to increasing fraction of $\mathrm{sp}^{2}$ carbons, which contribute to the $\mathrm{G}$ band but not to the $\mathrm{D}$ band ${ }^{13,14}$. According to Ref 14 , the $I_{\mathrm{D}} / I_{\mathrm{G}}$ ratio is $\sim 0.3$ for 7 -AGNR $(0.74 \mathrm{~nm})$ and $\sim 1.2$ for 14-AGNR. Thus, the larger $I_{\mathrm{D}} / I_{\mathrm{G}}$ ratio on AGNRs/Ag(111) suggests the coexistence of wider AGNRs such as 14-AGNRs or 21-AGNRs with 7-AGNRs. Besides these six modes, the spectrum exhibits a sharp peak at $\sim 2000 \mathrm{~cm}^{-1}$, as highlighted in the middle inset, originating from the localized width-independent mode generated by the vibration of the atoms at the edge of $\mathrm{AGNRs}^{15}$. Notably, only when the AGNRs are at least partially $\mathrm{H}$-free, that is, when $\mathrm{C} \equiv \mathrm{C}$ bonds exist at their armchair edges, can the localized mode be observed $^{15}$. This gives evidence for the dehydrogenation at the head sites and the formation of AGNRs with multiple integer widths of 14-, 21-AGNRs and so on.

The STM image inserted in the upper-right corner in Fig. 2a clearly shows the randomly distributed AGNRs with varying widths on $\operatorname{Ag}(111)$. The green rectangles highlight one 7-14-7-AGNR and one 14-21-AGNR heterojunction, where the $\pi$-conjugation at the junction interface is preserved. The line profiles taken perpendicularly across 7-, 14-, 21-, and 28-AGNRs axes reveal the corresponding widths of $\sim 1.5 \mathrm{~nm}, \sim 2.4 \mathrm{~nm}, \sim 3.3 \mathrm{~nm}$, and $\sim 4.2 \mathrm{~nm}$ respectively, and an apparent height of $\sim 0.19 \mathrm{~nm}$, as shown in Fig. 2b. These values vary slightly depending on tip conditions. For such AGNRs the widths measured are larger than those from the corresponding atomic structure models. For example, the model of 7-AGNR (Fig. 1b) shows a smaller width of $0.741 \mathrm{~nm}$. This could be attributed to the finite size of STM tip. The highly-resolved STM image taken at $77 \mathrm{~K}$ in Fig. $2 \mathrm{c}$ shows ESPs in 7- and 21-AGNRs, which arise from the intervalley electron backscattering at both armchair edges $^{10,11}$.

\section{Discussion}

We first discuss the two Raman peaks located at $\sim 2608 \mathrm{~cm}^{-1}$ and $\sim 2690 \mathrm{~cm}^{-1}$ in Fig. 2. A previous report shows Raman 2D peak splitting upon deposition of $\mathrm{Au}$ onto single layer graphene, which come from the graphene with and without $\mathrm{Au}$, and is not the case in this work ${ }^{16}$. Recent theoretical and experimental investigations reveal that the Raman 2D peak of single layer graphene splits into two peaks under homogeneous uniaxial strain and both redshift significantly depending on the direction and magnitude of the strain applied ${ }^{17-21}$. In particular, the shift rates are up to $-67.8 \mathrm{~cm}^{-1} / \%$ and $-26.0 \mathrm{~cm}^{-1} / \%$ for strain applied along the zigzag directions ${ }^{17}$. Accordingly, the average strain in the direction perpendicular to the AGNR axis is at least $2 \%$. In this work, the mismatch between AGNRs and $\mathrm{Ag}(111)$ may induce strain. However, we do not observe obvious deformation of AGNRs along its zigzag direction. Furthermore, uniaxial strain-induced redshift of the peak at $\sim 2690 \mathrm{~cm}^{-1}$ is not observed. We also note that the $2 \mathrm{D}$ peak of GNRs formed by CNT unzipping is observed at $\sim 2659 \mathrm{~cm}^{-16}$. Thus, strain-induced Raman $2 \mathrm{D}$ peak splitting is highly unlikely. We performed comparative Raman measurement on AGNRs of different widths. The Raman measurements on 7-AGNRs on $\mathrm{Au}(111)$ is shown in Fig. S1, where one peak is observed at $\sim 2603 \mathrm{~cm}^{-1}$ accompanied by a shoulder at $\sim 2685 \mathrm{~cm}^{-1}$. On Au surface, 7-AGNRs dominate, and the Raman peak at $\sim 2603 \mathrm{~cm}^{-1}$ is attributed to 7 -AGNRs. The shoulder at $2685 \mathrm{~cm}^{-1}$ appears to have the same origin as the peak at $\sim 2690 \mathrm{~cm}^{-1}$ for AGNRs on $\mathrm{Ag}(111)$. Wider AGNRs such as 14and 21-AGNRs are observed on $\operatorname{Ag}(111)$ compared to that on $\mathrm{Au}(111)$. Hence, from our empirical observations, we suggest that the peak at $\sim 2690 \mathrm{~cm}^{-1}$ is attributed to wider AGNRs. Further calculations are required to support this postulate.

Fig. 3 displays STM images of ESPs in 7-, 14-, and 21-AGNRs (left column) as well as the corresponding DFT simulations (right column), respectively. Figs. $3 a$ and $3 c$ are enlarged from Fig. 2c. ESPs in 7-AGNR form localized ring-like shapes with a $(\sqrt{3} \times \sqrt{3}) \mathrm{R} 30^{\circ}$ unit cell, in good agreement with the calculated charge density plots of the conduction bands of 7-AGNR (filled red part in left panel in Fig. 3a) ${ }^{7}$ and with our DFT simulations (right panel in Fig. 3a). Such ESPs are attributed to the electronic states in the vicinity of the armchair edge where the incident and reflected waves interfere with each other due to intervalley scattering ${ }^{10,11}$. A piece of ball and stick model of 7AGNR is superimposed in the STM image of Fig. 3a, with the red dots highlighting each centre of the ESPs. This suggests that ESPs centres at both armchair edges of 7-AGNR are located not at but between the benzene-ring edges. One edged benzene-ring and the location between two adjacent benzene-ring edges are highlighted in Fig. 1 b by a yellow hexagon and red arrows, respectively. ESPs in 21AGNR are similar to those in 7-AGNR, in a localized ring-like shape with a $(\sqrt{3} \times \sqrt{3}) \mathrm{R} 30^{\circ}$ unit cell, as marked by a red parallelogram. It is worth noting that there is a dislocation line parallel to the 21-AGNR axis, as highlighted by the green dash-dotted line. Given that 21AGNR has the same lateral mirror symmetry as 7-AGNR and that the armchair edges have the same scattering effect on the incident electrons, we extend the above-mentioned calculated charge density plots of the conduction bands from 7-AGNR to 21-AGNR. The red (blue) dots indicate the ESP centres due to electron backscattering at the lower (upper) edge, as shown in the overlaid 21-AGNR ball and stick model in the left panel in Fig. 3c. Using geometrical considerations, ESPs from opposite armchair edges will be misaligned in the direction $60^{\circ}$ off the lateral direction of 21-AGNR (parallel to the $\sqrt{3}$ 

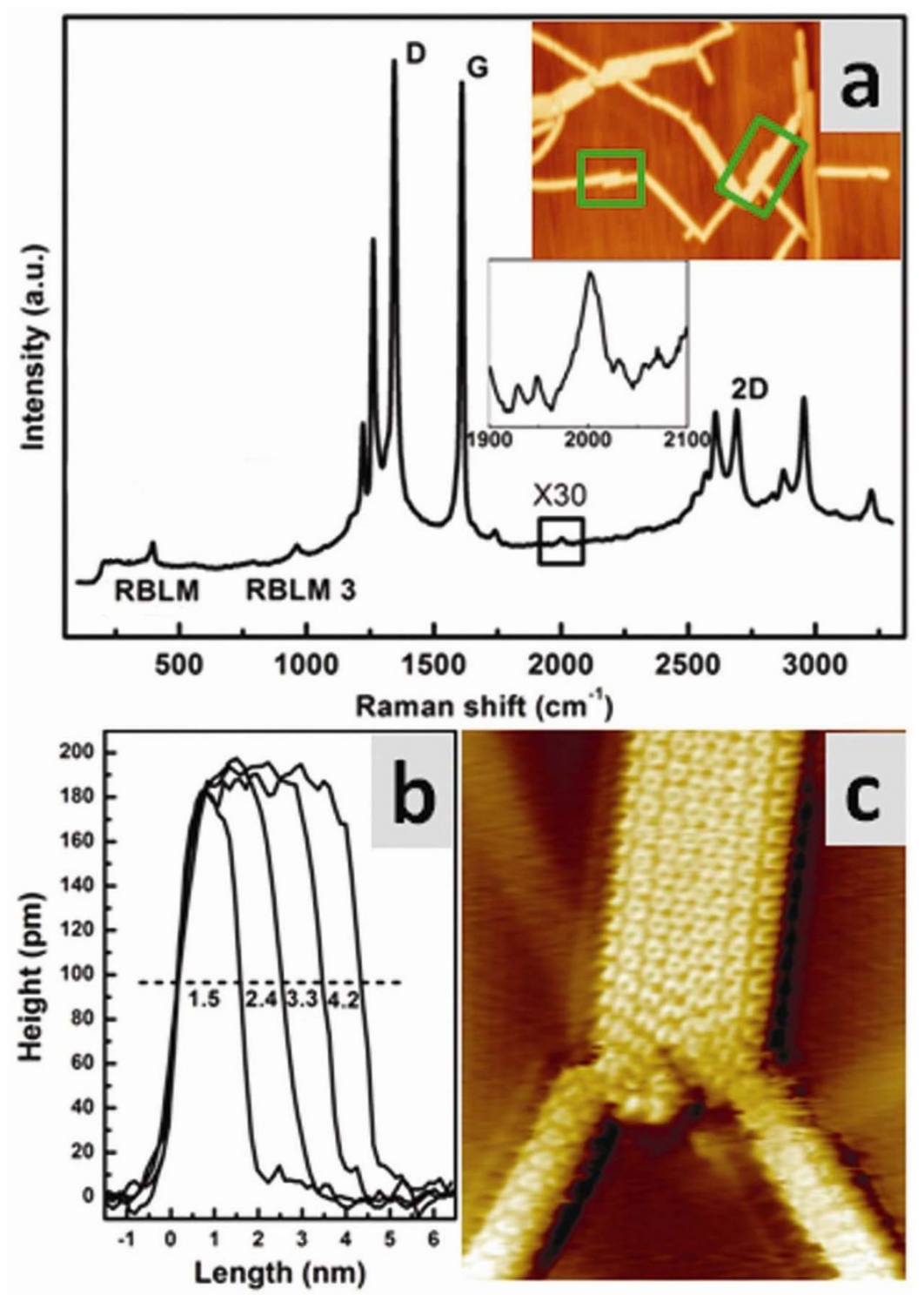

Figure $2 \mid$ a) Raman spectrum on AGNRs/Ag(11) under ambient conditions. Besides mode D (1350 $\left.\mathrm{cm}^{-1}\right)$ and mode $\mathrm{G}\left(1610 \mathrm{~cm}^{-1}\right)$, some other vibration modes appear due to the finite width and low symmetry of the nanoribbons, such as RBLM $\left(396 \mathrm{~cm}^{-1}\right), \mathrm{RBLM}\left(961 \mathrm{~cm}^{-1}\right)$. The peak at $2000 \mathrm{~cm}^{-1}$ as highlighted in the middle inset arises from the vibration mode of the C-atoms at the edge of AGNRs. The inserted typical STM image (70 nm $\times 40 \mathrm{~nm}, \mathrm{~V}_{\mathrm{T}}=-2.0 \mathrm{~V}$ ) at the upper-right corner shows straight AGNRs with varying widths. b) line-profiles taken perpendicularly across the 7-, 14-, 21-, and 28-AGNRs, respectively. c) STM image $\left(8 \mathrm{~nm} \times 10 \mathrm{~nm}, \mathrm{~V}_{\mathrm{T}}=-0.5 \mathrm{~V}\right)$ showing the electron scattering patterns in 7- (lower) and 21-AGNRs (upper). The 21-AGNR is imperfect at the lower end.

direction as indicated by the red parallelogram), which will result in the observed dislocation. This is consistent with the DFT simulations in the right panel in Fig. 3c. The dislocation shifts to a lower position at the left end of the AGNR because of defects (see Fig. 2c, the lower end of 21-AGNR).

ESPs in 14-AGNR are significantly different, as shown in the left panel in Fig. 3b. One-dimensional (1D) delocalized striped patterns were observed parallel to its axis with equal-spacing of $\sim 0.38 \mathrm{~nm}$, in good agreement with the Fermi wavelength of graphene of $3 a / 2=$ $0.37 \mathrm{~nm}$, where $a=0.246 \mathrm{~nm}$ is the lattice constant of graphene. Such ESPs arise from the intervalley electron backscattering at both armchair edges of metallic AGNRs ${ }^{3}$ and depend not on the electron energy but only on the edge structure, as observed at the armchair edge of epitaxial graphene on $\mathrm{SiC}(0001)^{22}$. A piece of ball and stick model of 14-AGNR is overlaid to show the registration between the ESPs and C chains. This model matches perfectly with the prediction $^{3}$ : the probability density for a state near the Dirac singularity disappears at $\mathrm{J}=3 i$ (i.e. $3,6,9,12 \ldots \ldots$ ), where $i$ is an integer, and $\mathrm{J}$ is introduced to mark the position of carbon-pair lines in AGNRs, as shown in Fig. 3b. The good match with the DFT calculations in the right panel of Fig. $3 \mathrm{~b}$ confirms the $1 \mathrm{D}$ metallic nature of 14-AGNR due to quantum confinement effects ${ }^{3}$.

In order to acquire more detailed information about the electronic structures of AGNRs, $d \mathrm{I} / d \mathrm{~V}$ measurements were performed at $4.7 \mathrm{~K}$. These conductance measurements are directly proportional to the LDOS within the Tersoff-Harmann approximation ${ }^{23-26}$. The $d \mathrm{I} / d \mathrm{~V}$ spectra in Figs. 4, 5, and S2 reveal the electronic properties of such AGNRs. Fig. 4 summarizes the typical $d \mathrm{I} / d \mathrm{~V}$ spectra from the centres of three neighbouring AGNRs, which are shown in the inserted STM image. The bottom spectra taken from clean $\mathrm{Ag}(111)$ terrace clearly show the surface states at $\sim 66 \mathrm{mV}$ below Fermi energy level $\left(\mathrm{E}_{\mathrm{F}}, 0 \mathrm{~V}\right.$ in the spectrum), as marked by a short cyan solid line, verifying the good tip conditions. The position of $A G N R E_{F}$ as indicated by the green dash-dotted line is shifted toward their unoccupied bands. This 


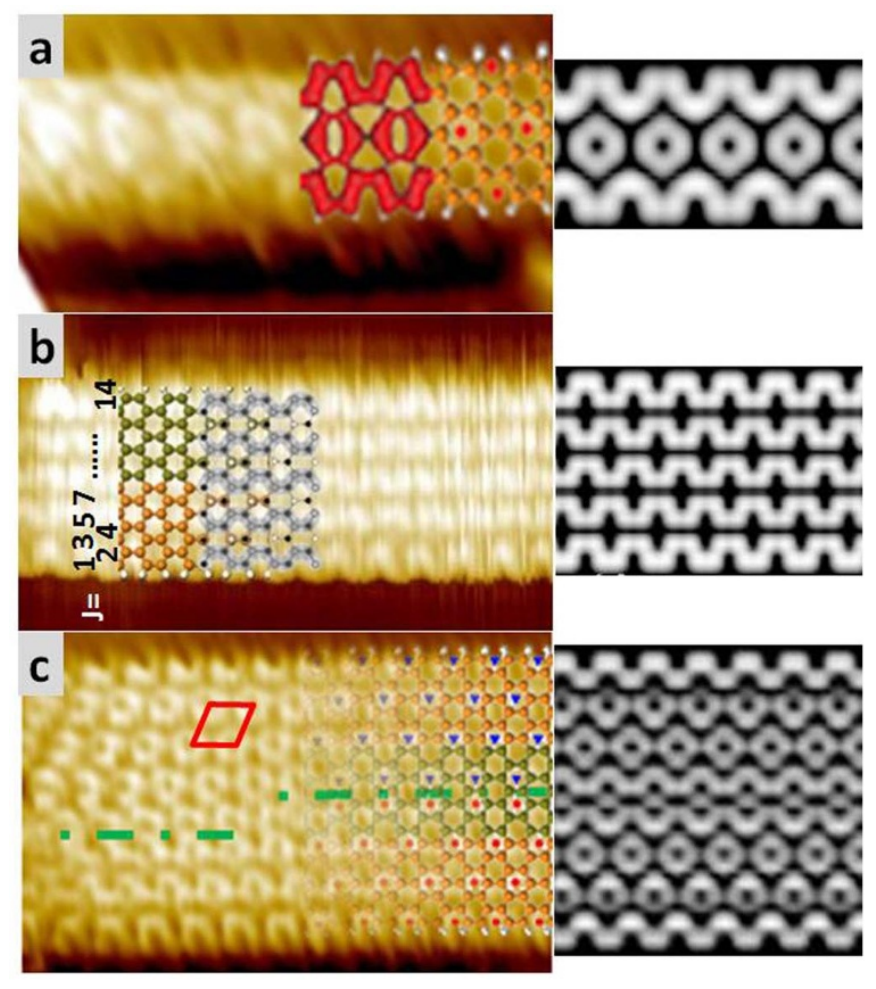

Figure $3 \mid$ ESPs (left column, a: $3.5 \mathrm{~nm} \times 2 \mathrm{~nm}, \mathrm{~V}_{\mathrm{T}}=-0.5 \mathrm{~V}$; b: $5 \mathrm{~nm} \times$ $3 \mathrm{~nm}, \mathrm{~V}_{\mathrm{T}}=-0.5 \mathrm{~V}$; c: $\left.5.5 \mathrm{~nm} \times 3.5 \mathrm{~nm}, \mathrm{~V}_{\mathrm{T}}=-0.5 \mathrm{~V}\right)$ and corresponding DFT simulations (right column) in 7-, 14-, and 21-

AGNRs. Parameter $J$ in panel $b$ is an integer that marks the position of carbon-pair lines in AGNRs. The red parallelogram in Fig $3 \mathrm{c}$ marks the $(\sqrt{3}$ $\times \sqrt{3}) \mathrm{R} 30^{\circ}$ unit cell, the green dash-dotted line for dislocation, which shifts due to the defects at left end (see Fig. 2c). Red and blue dots: ESP centres.

is attributed to the smaller work function of $\mathrm{Ag}(111)(\sim 4.4 \mathrm{eV})$ and a hybridization between Ag $d$ band and graphene $2 p^{27,28}$. The spectra exhibit a sharp peak at $\sim-240 \mathrm{mV}$ for 21 -AGNR, a broad peak at $\sim-400 \mathrm{mV}$ for 14 -AGNR and a broader peak at $\sim-800 \mathrm{mV}$ for 7AGNR, as marked by green arrows, respectively.

Fig. 5 shows $\mathrm{dI} / \mathrm{dV}$ spectra acquired perpendicularly across AGNRs. In each panel, the black spectra were taken from the adjacent $\operatorname{Ag}(111)$ surface, while the red (blue) ones are from the AGNRs edges (centre) at equal spacing. The inserted differential STM images show the typical AGNRs respectively. For 7-AGNR, $d \mathrm{I} / d \mathrm{~V}$ spectra exhibit a broad peak at $\sim-850 \mathrm{mV}$ at the edges and is almost featureless in the centre, as shown in Fig. 5a. Taking the substrate influence into account ${ }^{28}$, it is in consistence with the recent report by Ruffieux et al. ${ }^{29}$ that the conduction band minimum of $\mathrm{Au}(111)$ supported 7-AGNR is located at $1.6 \mathrm{eV}$ above $\mathrm{E}_{\mathrm{F}}$ at its armchair edges. It is also in good agreement with the recent report by Koch M. that the HOMO and LUMO of Au(111)-supported 7-AGNRs delocalized along their armchair edges with an energy gap of $2.7 \mathrm{eV}^{30}$. Detailed inspection reveals that the unoccupied bands of 7-AGNR is shifted below $\mathrm{E}_{\mathrm{F}}$ and thus becomes partially occupied, indicating a weak chemisorption nature ${ }^{31}$. On the contrary, while the featureless spectra are partially occupied at the edges, those in centre remain unoccupied and exhibit a broad (sharp) peak at $\sim-380 \mathrm{mV}$ $(\sim-240 \mathrm{mV})$ for 14-AGNR (21-AGNR), as highlighted by solid green lines in Fig. 5 respectively. The spatially resolved spectra indicate that the electronic structures are heavily influenced when AGNRs adsorbed on $\operatorname{Ag}(111)$. For $0 \mathrm{~V}<\mathrm{V}_{\mathrm{T}}<1.0 \mathrm{~V}$, the LDOS of all the AGNRs are finite and similar to that of exposed $\mathrm{Ag}(111)$ (for the case of 21-AGNR see Fig. S2). This suggests that in the energy gap of AGNRs the local density of states from underlying $\mathrm{Ag}(111)$ could

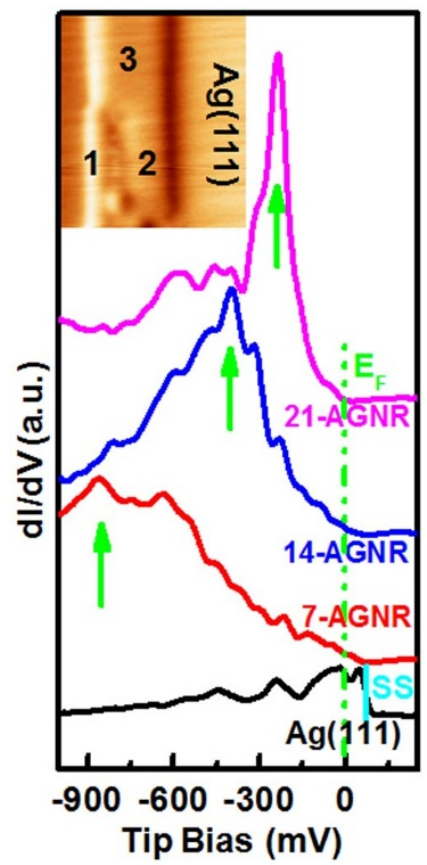

Figure 4 | STS data showing the unoccupied states of clean $\operatorname{Ag}(111)$ and centers of 7-, 14- and 21-AGNRs, as marked on the inserted STM image $\left(7 \mathrm{~nm} \times 8 \mathrm{~nm}, \mathrm{~V}_{\mathrm{T}}=1.5 \mathrm{~V}\right)$ as 1,2 and 3 respectively, clearly show that electronic properties of AGNRs are significantly related to their widths. Offset vertically for clarity. The green dash-dotted line indicates $E_{F}$ and the short cyan solid line for the surface states of clean $\mathrm{Ag}(111)$ at $\sim 66 \mathrm{mV}$. The green arrows indicate the typical peaks in STS spectra.

not be excluded in STS measurements. Due to the charge transfer between AGNRs and $\operatorname{Ag}(111)$, the corresponding valence band maximum of AGNRs shifts out of the measurement range and could not be detected with our experimental settings. Applying Ruffieux's method $^{29}$, the energy gap of semiconducting 7-AGNRs is deduced to be over $1.8 \mathrm{eV}$, in good agreement with recent experimental reports $^{29,30,32,33}$. For 21-AGNRs, the energy gap is over $1.2 \mathrm{eV}$. For metallic 14-AGNRs, the observed peak at $-380 \mathrm{meV}$ might be the first Van Hove singularity (VHS) at the unoccupied states, which is broadened due to the hybridization with $\operatorname{Ag}(111)^{28,34}$. The first VHS gap of metallic 14-AGNR is calculated to be close to $2 \mathrm{eV}^{35}$, and is also out of the measurement range. Further experiments and simulations are required to accurately measure the energy gaps.

For heterojunctions such as 7-14-AGNRs, 14-21-AGNRs, the spectra taken along the ribbons exhibit the previously mentioned characteristics of each AGNR when measured in their respective regions (Fig. 4). The change in spectra across the boundary $(<$ $1 \mathrm{~nm}$ ) is very sharp, having no transitional spectra. This is similar to the case of metallic intramolecular heterojunctions in $\mathrm{CNT}^{36}$, confirming that the electronic properties of AGNRs are significantly width-dependent.

Why do AGNRs with varying widths form on $\mathrm{Ag}(111)$ ? To answer this question, a submonolayer DBBA on $\mathrm{Ag}(111)$ annealed at $\sim 450 \mathrm{~K}$ for $30 \mathrm{~min}$ was investigated. Unexpectedly, instead of anthracene polymers, isolated planar products are observed, as shown in Fig. 6, indicating that dehydrogenation has taken place for DBBA monomers on $\mathrm{Ag}(111)$ at this stage ${ }^{37}$. The carbon backbone of an isolated molecular product is inserted at the upper-right corner of Fig. 6a. More theoretical simulations are required to determine how many hydrogen atoms dissociate from one DBBA molecule and its final configuration. Two such structural units are tentatively superimposed on the high resolution STM image in Fig. 6 d. Fig. $6 \mathrm{~b}$ shows the line profile taken along the white line in 


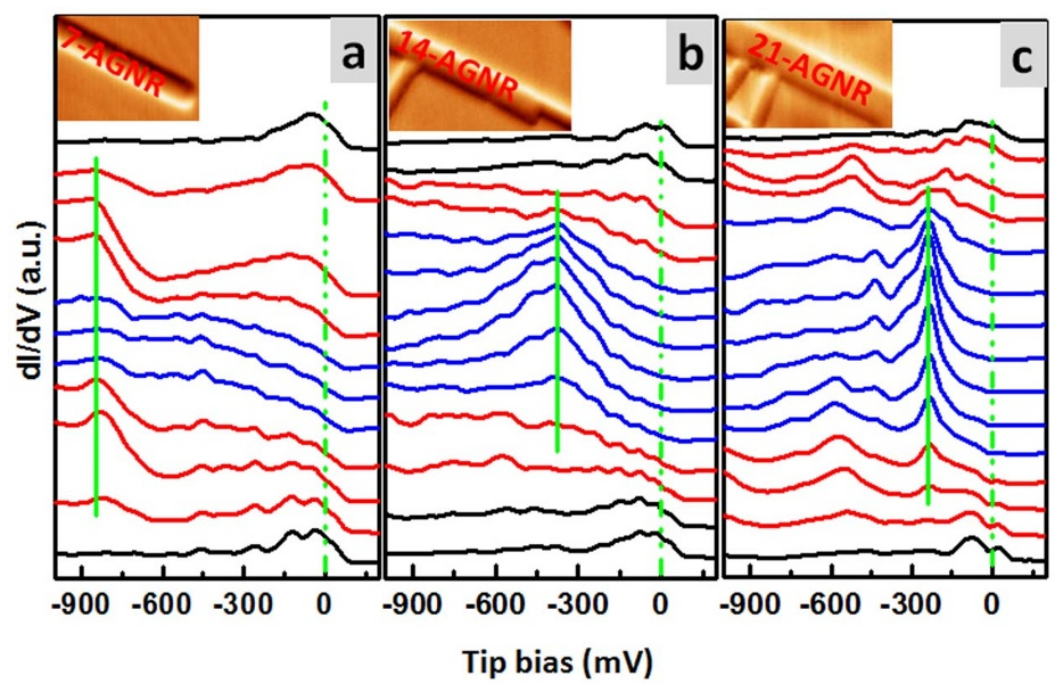

Figure 5 STS spectra along the lines across 7-, 14- and 21-AGNRs perpendicularly, respectively. Offset vertically for clarity. The green dash-dotted lines indicate the Fermi level, the green lines for the main peaks.

Fig. 6a, and Fig. 6c shows the corresponding fast Fourier transform (FFT) pattern from Fig. 6a, revealing that isolated planar molecular products form a hexagonal arrangement with a nearest neighbour distance of $1.5 \pm 0.1 \mathrm{~nm}$. This is much larger than that of $\sim 1.15 \mathrm{~nm}$ for monolayer coronene on $\operatorname{Ag}(111)^{38}$, indicating a strong adsorbatesubstrate interaction at $77 \mathrm{~K}$. Some short rod-like features of two or three connected intermediate products are observed in the area enclosed by white dash-dotted curve, indicating that such planar products act as intermediates for AGNRs. The growth mechanism of AGNRs on $\mathrm{Ag}(111)$ is proposed in Fig. S3, which is significantly different with that on $\mathrm{Au}(111)^{7}$. It may be related to the different catalytic reactivities of $\mathrm{Au}(111)$ and $\mathrm{Ag}(111)$, which induce different self-assembly behaviour of DBBA at room temperature, as shown in Fig. S4.

In summary, we have succeeded in fabricating ultra-narrow atomically precise 7-, 14- and 21-AGNRs and observed their associated ESPs using LT-STM. The observed ESPs are in good agreement with theoretical predictions. The $d \mathrm{I} / d \mathrm{~V}$ spectra reveal the width-dependent electronic properties of the AGNRs. For the next step of our investigation, we plan to investigate the electronic properties of such AGNRs on insulating thin films. We expect that these initial investigations on the electronic properties of such AGNRs give us a better understanding of this extraordinary class of materials and help advance theoretical predictions on systems such as intraribbon quantum dots ${ }^{39}$, GNR switches ${ }^{40}$ and magnetic devices based on specific GNR edge states ${ }^{41}$.

\section{Methods}

Synthesis of AGNRs. The clean $\operatorname{Ag}(111)$ surface was achieved in situ by repeated cycles of Ar+ sputtering and subsequent annealing to $\sim 750 \mathrm{~K}$. DBBA (97\%, St-Jean Photochemical Inc.) was evaporated from a K-cell (MBE-Komponenten, Germany) at a deposition rate of $\sim 0.01 \mathrm{ML} / \mathrm{min}$ (ML: monolayer) at normal incidence onto $\mathrm{Ag}(111)$ at room temperature. After deposition, the sample was annealed at $\sim 450 \mathrm{~K}$ and $\sim 650 \mathrm{~K}$ for for $30 \mathrm{mins}$, respectively.

STM/STS measurements. Sample preparation and STM measurements were carried out in a custom-built multi-chamber ultra-high vacuum (UHV) system housing an

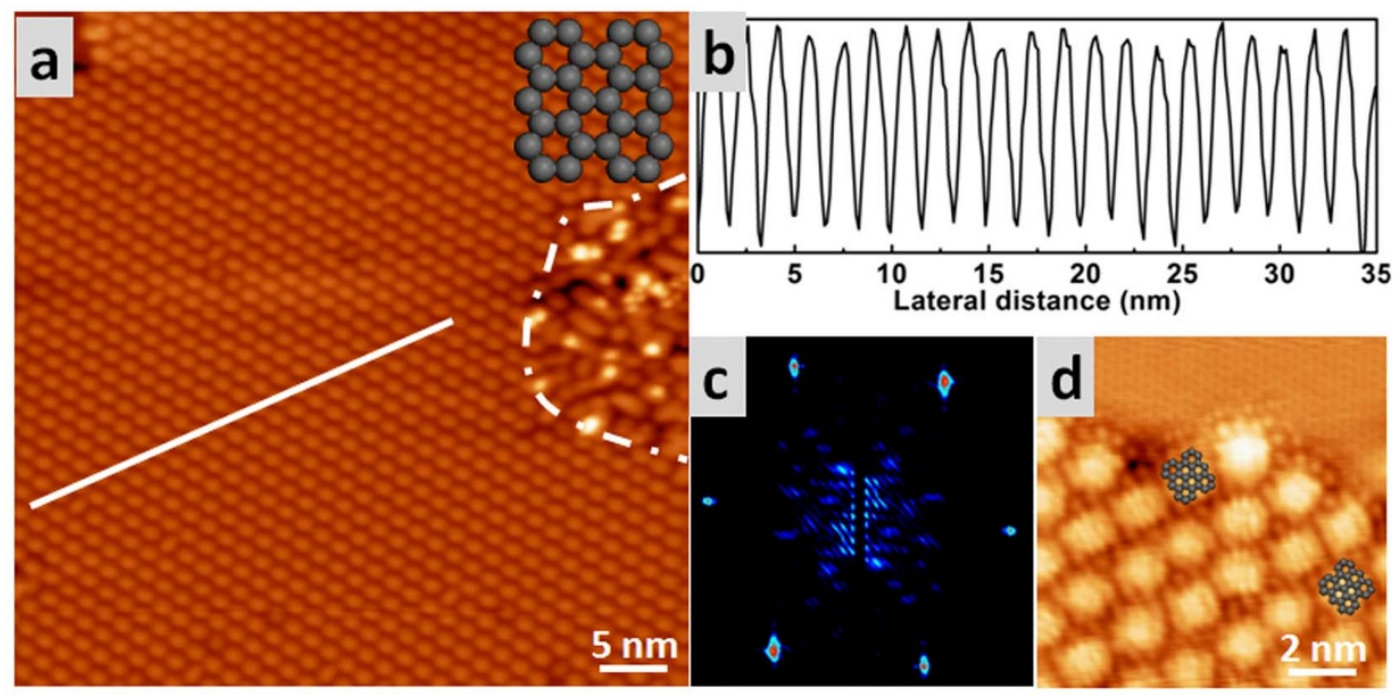

Figure 6 Dehydrogenation of DBBA on $\mathrm{Ag}(111)$ at $\sim 450 \mathrm{~K}$. a) STM image $\left(\mathrm{V}_{\mathrm{T}}=-1.0 \mathrm{~V}\right)$ showing that most of the products are isolated in a hexagonal arrangement, as revealed by the corresponding FFT pattern in panel c; while some are connected together as short rods, as highlighted by the white dash-dotted curve. The carbon backbone of the isolated product is inserted at the upper right corner. b) the line-profile taken along the white line in panel a, showing an average intermolecular distance of $1.5 \pm 0.1 \mathrm{~nm}$. d) zoomed-in STM image $\left(\mathrm{V}_{\mathrm{T}}=-0.01 \mathrm{~V}\right)$ showing the isolated product lies flat on $\mathrm{Ag}(111)$. Two molecular unit structures are superimposed. Such planar products are believed to be the intermediates for AGNRs in varying widths. 
Omicron LT-STM in the analysis chamber with a base pressure better than $6.0 \times 10^{-11}$ mbar. All the STM images were recorded in constant current mode using chemically etched tungsten (W) tips. To reduce the thermal drift and noise level, differential conductance $(d \mathrm{I} / d \mathrm{~V})$ measurements were performed at $\sim 4.7 \mathrm{~K}$ in an open feedback loop mode using the lock-in technique with frequency $625 \mathrm{~Hz}$ and ac modulation of $20 \mathrm{meV}$ and with set-point parameters of $\mathrm{V}_{\mathrm{T}}=100 \mathrm{mV}$ and $\mathrm{I}=200 \mathrm{pA}$.

Raman measurements. Raman spectra were recorded with a Renishaw 1000 Raman Microscope at ambient. The excitation source was a $514 \mathrm{~nm}$ laser with power below $20 \mathrm{~mW}$ to avoid laser induced surface heating. The laser spot size was around $10 \mu \mathrm{m}$.

Simulation of the ESPs. Simulations of electron scattering patterns (ESPs) of pristine graphene nanoribbon were obtained by performing $a b$-initio pseudopotential density functional theory calculations. In Tersoff and Hamann approximation ${ }^{17}$, the tunneling current density between the STM tip and the sample is proportional to the local density of states (LDOS)

$$
\rho\left(V_{\text {bias }}\right)=\int_{E_{F}-V_{\text {bias }}}^{E_{F}} \sum_{i}^{\text {levels }} \sum_{\bar{\kappa} \in B Z}\left|\Phi_{i},{ }_{\bar{\kappa}}\right|^{2} \delta\left(E_{i}, \bar{\kappa}-E\right) d E .
$$

$\Phi_{i, \bar{\kappa}}$ is the wave function of pristine graphene nanoribbon. When a small bias voltage $V_{\text {bias }}$ is applied, the numerical integration is carried out on the associated eigenvalues within this energy range below the Fermi level. This provides occupied (unoccupied) electronic states when a negative (positive) bias voltage is applied. The exchangecorrelation functionals are described by the generalized gradient approximation (GGA) derived Perdew-Burke-Ernzerhof (PBE) approach ${ }^{18}$. The calculations were performed by using the SIESTA code ${ }^{19}$, together with a double- $\zeta$ polarized basis-set ${ }^{20}$, a real space mesh cutoff of 400 Ryd and norm conversing pseudopotential. The slab models include a vacuum thickness of $10 \AA$ to reduce the influence of periodic images along the direction perpendicular to the GNR plane. The total energies converged well with respect to the sufficiently large k-point sampling of $90 \times 1 \times 1$ in the Brillouin zone. The electronic structure calculations were carried out using fully relaxed structures where the structural optimization on the GNR atomic degrees of freedom was achieved until the residual forces on each atom are smaller than $0.01 \mathrm{eV} / \AA$.

1. Nakada, K., Fujita, M., Dresselhaus, G. \& Dresselhaus, M. S. Edge State in Graphene Ribbons: Nanometer Size Effect and Edge Shape Dependence. Phys. Rev. B 54, 17954-17961 (1996).

2. Son, Y. W., Cohen, M. L. \& Louie, S. G. Energy Gaps in Graphene Nanoribbons. Phys. Rev. Lett. 97, 216803 (2006).

3. Sasaki, K., Wakabayashi, K. \& Enoki, T. Electron Wave Function in Armchair Graphene Nanoribbons. J. Phys. Soc. Jpn. 80, 044710 (2011).

4. Han, M. Y., Ozyilmaz, B., Zhang, Y. \& Kim, P. Energy Band-Bap Engineering of Graphene Nanoribbons. Phys. Rev. Lett. 98, 206805 (2007).

5. Tapaszto, L., Dobrik, G., Lambin, P. \& Biro, L. P. Tailoring the Atomic Structure of Graphene Nanoribbons by Scanning Tunneling Microscope Lithography. Nat. Nanotechnol. 3, 397-401 (2008).

6. Jiao, L. Y., Zhang, L., Wang, X. R., Diankov, G. \& Dai, H. J. Narrow Graphene Nanoribbons from Carbon Nanotubes. Nature 458, 877-880 (2009).

7. Cai, J. M., Ruffieux, P., Jaafar, R., Bieri, M., Braun, T., Blankenburg, S., Muoth, M., Seitsonen, A. P., Saleh, M., Feng, X. L., Mullen, K. \& Fasel, R. Atomically Precise Bottom-Up Fabrication of Graphene Nanoribbons. Nature 466, 470-473 (2010).

8. Blankenburg, S., Cau, J. M., Ruffieux, P., Jaafar, R., Passerone, D., Feng, X. L., Mullen, K., Fasel, R. \& Pignedoli, C. S. Intraribbon Heterojunction Formation in Ultranarrow Graphene Nanoribbons. ACS Nano 6, 2020-2025 (2012).

9. Bjork, J., Stafstrom, S. \& Hanke, F. Zipping Up: Cooperativity Drives the Synthesis of Graphene Nanoribbons. J. Am. Chem. Soc. 133, 14884-14887 (2011).

10. Niimi, Y., Matsui, T., Kambara, H., Tagami, K., Tsukada, M. \& Fukuyama, H. Scanning Tunneling Microscopy and Spectroscopy of the Electronic Local Density of States of Graphite Surfaces near Monoatomic Step Edges. Phys. Rev. B 73, 085421 (2006).

11. Sakai, K., Takai, K., Fukui, K., Nakanishi, T. \& Enoki, T. Honeycomb Superperiodic Pattern and its Fine Structure near the Armchair Edge of Graphene Observed by Low-Temperature Scanning Tunneling Microscopy. Phys. Rev. B 81, 235417 (2010).

12. Saito, R., Furukawa, M., Dresselhaus, G. \& Dresselhaus, M. S. Raman Spectra of Graphene Ribbons. J. Phys: Condens. Matter 22, 334203 (2010).

13. Ryu, S., Maultzsch, J., Han, M. Y., Kim, P. \& Brus, L. E. Raman Spectroscopy of Lithographically Patterned Graphene Nanoribbons. ACS Nano 5, 4123-4130 (2011)

14. Ferrari, A. C. \& Robertson, J. Interpretation of Raman Spectra of Disordered and Amorphous Carbon. Phys. Rev. B 61, 14095-14107 (2000).

15. Zhou, J. \& Dong, J. M. Vibrational Property and Raman Spectrum of Carbon Nanoribbon. Appl. Phys. Lett. 91, 173108 (2007).

16. Entani, S., Sakai, S., Matsumoto, Y., Naramoto, H., Hao, T. \& Maeda, Y. Interface Properties of Metal/Graphene Heterostructures Studied by Micro-Raman Spectroscopy. J. Phys. Chem. C. 114, 20042-20048 (2010).

17. Yoon, D., Son, Y. W. \& Cheong, H. Strain-Dependent Splitting of the DoubleResonance Raman Scattering Band in Graphene. Phys. Rev. Lett. 106, 155502 (2011).
18. Huang, M. Y., Yan, H., Heinz, T. F. \& Hone, J. Probing Strain-induced Electronic Structure Change in Graphene by Raman Spectroscopy. Nano Lett. 10, 4074-4079 (2010).

19. Mohr, M., Maultzsch, J. \& Thomsen, C. Splitting of the Raman 2D Band of Graphene subjected to Strain. Phys. Rev. B 82, 201409(R) (2010).

20. Frank, O., Mohr, M., Maultzsch, J., Thomsen, C., Riaz, I., Jalil, R., Novoselov, K. S., Tsoukleri, G., Parthenios, J., Papagelis, K., Kavan, L. \& Galiotis, C. Raman 2DBand Splitting in Graphene: Theory and Experiment. ACS Nano 5, 2231-2239 (2011).

21. Frank, O., Tsoukleri, G., Parthenios, J., Papagelis, K., Riaz, I., Jalil, R., Novoselov, K. S. \& Galiotis, C. Compression Behavior of Single-Layer Graphenes. ACS Nano 4, 3131-3138 (2010).

22. Yang, H., Mayne, A. J., Boucherit, M., Comtet, G., Dujardin, G. \& Kuk, Y. Quantum Interference Channeling at Graphene Edges. Nano Lett. 10, 943-947 (2010).

23. Tersoff, J. \& Hamann, D. R. Theory and Application for the Scanning Tunneling Microscope. Phys. Rev. Lett. 50, 1998-2001 (1983).

24. Perdew, J. P., Burke, K. \& Ernzerhof, M. Generalized Gradient Approximation Made Simple. Phys. Rev. Lett. 77, 3865-3868 (1996).

25. Sánchez-Portal, D., Ordejón, P., Artacho, E. \& Soler, J. M. Density Functional Method for Very Large Systems with LCAO Basis Sets. Int. J. Quantum Chem. 65, 453-461 (1997)

26. Junquera, J., Paz, Ó., Sánchez-Portal, D. \& Artacho, E. Numerical Atomic Orbitals for Linear-Scaling Calculations. Phys. Rev. B 64, 235111-235119 (2001).

27. Wildoer, J. W. G., Venema, L. C., Rinzler, A. G., Smalley, R. E. \& Dekker, C. Electronic Structure of Atomically Resolved Carbon Nanotubes. Nautre 391, 59-62 (1998).

28. Clair, S., Kim, Y. \& Kawai, M. Energy Level Alignment of Single-walled Carbon Nanotubes on Metal Surfaces. Phys. Rev. B 83, 245422 (2011).

29. Ruffieux, P., Cai, J. M., Plumb, N. C., Patthey, L., Prezzi, D., Ferretti, A., Molinari, E., Feng, X. L., Mullen, K., Pignedoli, C. A. \& Fasel, R. Electronic Structure of Atomically Precise Graphene Nanoribbons. Acs Nano, 6, 6930-6935 (2012).

30. Koch, M., Ample, F., Joachim, C. \& Grill, L. Voltage-dependent Conductance of a Single Graphene Nanoribbon. Nat. Nanotechnol. 7, 713-717 (2012).

31. Norskov, J. K. Chemisorption on Metal Surface. Rep. Prog. Phys. 53, 1253-1295 (1990).

32. Linden, S., Zhang, D., Timmer, A., Aghdassi, N., Franke, J. H., Zhang, H., Feng, X., Mullen, K., Fuchs, H., Chi, L. \& Zacharias, H. Electronic Structure of Specially Aligned Graphene Nanoribbons on Au(788). Phys. Rev. Lett. 108, 216801 (2012).

33. Bronner, C., Leyssner, F., Stremlau, S., Utecht, M., Saalfrank, P., Klamroth, T. \& Tegeder, P. Phys. Rev. B 86, 085444 (2012).

34. Odom, T. W., Huang, J. L., Kim, P. \& Lieber, C. M. Atomic Structure and Electronic Properties of Single-Walled Carbon Nanotubes. Nature 391, 62-64 (1998).

35. Long, M. Q., Tang, L., Wang, D., Wang, L. J. \& Shuai, Z. G. Theoretical Predictions of Size-dependent Carrier Mobility and Polarity in Graphene. J. Am. Chem. Soc. 131, 17728-17729 (2009).

36. Ouyang, M., Huang, J. L., Cheung, C. L. \& Lieber, C. M. Atomically Resolved Single-Walled Carbon Nanotube Intramolecular Junctions. Science 291, 97-100 (2002).

37. Treier, M., Pignedoli, C. A., Laino, T., Rieger, R., Müllen, K., Passerone, D. \& Fasel, R. Surface-assisted Cyclodehydrogenation Provides a Synthetic Route towards Easily Processable and Chemically Tailored Nanographene. Nature Chem. 3, 61-67 (2011)

38. Lackinger, M., Griessl, S., Heckl, W. M. \& Hietschold, M. Coronene on Ag(111) Investigated by LEED and STM in UHV. J. Phys. Chem. B 106, 4482-4485 (2002).

39. Prezzi, D., Varsano, D., Ruini, A. \& Molinari, E. Quantum-Dot States and Optical Excitations on Edge-Modulated Graphene Nanoribbons. Phys. Rev. B 84, 041401 (2011).

40. Shi, Q. W., Wang, Z. F., Li, Q. X. \& Yang, J. L. Chiral Selective Tunneling Induced Graphene Nanoribbon Switch. Front. Phys. China 4, 373-377 (2009).

41. Son, Y. W., Cohen, M. L. \& Louie, S. G. Half-Metallic Graphene Nanoribbons. Nature 444, 347-349 (2006).

\section{Acknowledgements}

We acknowledge the financial support from NRF-CRP grants R-143-000-360-281: "Graphene and Related Materials and Devices" and R-144-000-295-281: "Novel 2D materials with tailored properties - beyond graphene". Dr Dacheng Wei thanks the support from Lee Kuan Yew Post Doctoral Fellowship Grant (R-144-000-263-112).

\section{Author contributions}

H.H., D.C.W. and A.T.S.W. planned the experiments. H.H. and S.L.W. collected and analyzed the data. J.T.S. and Y.P.F. performed the DFT calculations. H.H wrote the manuscript. A.T.S.W. supervised the project. A.H.C.N. provided theoretical insights and obtained funding for the project. All authors are involved in the revision of the manuscript 


\section{Additional information}

Supplementary information accompanies this paper at http://www.nature.com/ scientificreports

Competing financial interests: The authors declare no competing financial interests. License: This work is licensed under a Creative Commons
Attribution-NonCommercial-NoDerivs 3.0 Unported License. To view a copy of this license, visit http://creativecommons.org/licenses/by-nc-nd/3.0/

How to cite this article: Huang, H. et al. Spatially Resolved Electronic Structures of Atomically Precise Armchair Graphene Nanoribbons. Sci. Rep. 2, 983; DOI:10.1038/ srep00983 (2012). 